\title{
Synthesis of Nanocellulose from Coir Pith
}

\author{
Subha P.V ${ }^{1}$, Anita Das Rabindranath ${ }^{2}$
}

\begin{abstract}
Coir pith is lignocellulosic in nature and abundantly available by-product of the coir industry. It is a pollutant as it is resistant to biodegradation in the environment. It is mainly composed of cellulose (28\%), hemi cellulose $(9.5 \%)$ and lignin $(31 \%)$. In this study, cellulose was extracted from the coir pith, and treated generally for the production of nanocellulose. Cellulose is proved to be a versatile material due to its unique chemical structure, which provides a superior platform for several new biomaterials. Nanophase materials are prepared from inorganic materials and hardly any are reported from natural products. When particles are made up to nano size their reactivity increases. FT-IR, TGA, XRD and SEM characterized the extracted cellulose and nanocellulose. The hydrolyzed nanocellulose was found to be the mixed crystalline forms of cellulose I and II. Conversion of this lignocelluloscic biomass to nanocellulose will lead to the preparation of new-nano composites materials for biomedical applications.
\end{abstract}

Keywords: Coir pith, Crystalline, Biomaterials, Lignocellulosic, Nanophase, Nanocellulose

${ }^{1}$ CSIR-National Institute for Interdisciplinary Science and Technology (NIIST), Thiruvanathapuram, India. Email: subhapanampillil@gmail.com

${ }^{2}$ Corresponding author, Senior Scientific Officer, Central Coir Research Institute, P.O. - Kalavoor, Dist.Alleppey, Kerala-688 522, India.

Tel.: +91 9446488480; Email. anitadas30@ gmail.com 


\section{Introduction}

Nanophase materials, the miracles of the century have gained wide interest in recent years. Generally nanophase materials are prepared from inorganic materials and hardly any are reported from natural products. When particles are made up to nano size their reactivity increases. Properties of which depend strongly on their dimensions. It has variety of unique spectroscopic, electronic and chemical properties arising from their small sizes and high surface/ volume ratios.

Cellulose is the most ubiquitous and renewable natural resource in the biosphere. It has a complex, multilevel supra molecular structure and is a linear polymer consisting of $\mathrm{D}$ glucose subunits linked to each other by $\beta$-(I, 4)glycosidic bonds. The repeating unit of cellulose is cellobiose. In recent times, the demand for bio-based materials with renewable, low-cost and sustainable properties has increased. Cellulose-based materials can potentially provide products to meet this demand. In addition, the chemical modification of cellulosebased materials could be developed to meet the requirements set for environmental legislation. Cellulose is proved to be a versatile material due to its unique chemical structure, which provides a superior platform for several new biomaterials.

Cellulose has various potential uses within industries. Consequently, it has created an important focus for researcher's interest. Generation of bio based products and bio energy from such substances is important for the development of humans. On this field, cellulose production from natural fibers has become really momentous. In this work, cellulose was extracted from coir pith. Coir pith is lignocellulosic in nature and abundantly available. Coir pith is obtained from coconut husk during the extraction of coir fiber and is mainly composed of cellulose $(26.04 \%)$, hemi cellulose $(9.5 \%)$ and lignin $(31 \%)$. The cellulose polymers are linked together by hydrogen and vanderwaal bonds, which cause the cellulose to be packed in to micro fibrils. Cellulose in biomass is present in both crystalline and amorphous form. Hemi cellulose is linked to the micro fibrils by hydrogen bonds and is either homopolymers or a hetropolymers with short branches linked by $\beta$-(1,4)-glycosidic bonds and occasionally by $\beta-(1,3)$-glycosidic bonds. Lignin is a complex, large molecular structure containing cross-linked polymers of phenolic monomers, which are formed by the polymerization of three types of monomers: pCoumaryl (p-hydroxy phenyl propanol), Coniferyl (guaiacyl propanol) and Synapyl alcohols (syringal alcohol).

Coir pith obtained from the coconut husk is a pollutant as it is resistant to biodegradation in the environment. In this work, cellulose was extracted from the coir pith, and treated for production of nanocellulose. Cellulose nano crystals (CNs) are the fundamental constitutive polymeric motifs of macroscopic cellulose-based fibers whose sheer volume dwarfs any known natural or synthetic biomaterial. The main process for the isolation of nanocellulose from cellulose fiber is based on acid hydrolysis. Disordered or Para crystalline regions of cellulose are preferentially hydrolyzed, whereas crystalline regions that have a higher resistance to attack remain intact. Production of nanocellulose consists of subjecting cellulosic material to strong acid hydrolysis under strictly controlled conditions of temperature and time. If the nanocellulose is prepared by the hydrolysis using hydrochloric acid, their ability to disperse is limited and their aqueous suspension tends to flocculate. When sulphuric acid is used as a hydrolyzing agent, it reacts with the surface hydroxyl groups of cellulose to yield charged sulfate esters that promote dispersion of the CNS in water. However, the introduction of charged sulfate groups compromises the thermal stability of the nanocrystals. With respect to the morphology of the particles, a combination of both sulphuric acid and hydrochloric acids during hydrolysis appears to generate spherical CNs instead of rod- like nano crystals when carried out under ultrasonic treatment.

\section{Materials and Methods}

Coir pith collected from coir fiber extraction in and around, Alleppey was used as a raw material. Sodium chlorite $\mathrm{AR}\left(\mathrm{NaClO}_{2}\right)$, 
Sodium acetate, acetic acid and $\mathrm{NaOH}$ were used for the extraction of cellulose from coir pith. Dimethyl Sulphoxide (DMSO) and $\mathrm{NaOH}$ were used for pre-treatment of cellulose and inorganic acids such as sulphuric and hydrochloric acid were used for hydrolysis which was carried out using ultrasonic apparatus (Vibrionics, Japan).

\section{Cellulose extraction}

Raw coir pith was treated with Sodium chlorite $\left(\mathrm{NaClO}_{2}\right): 0.7 \% \quad \mathrm{w} / \mathrm{v}$ to yield holocellulose ( $\beta$-cellulose themicellulose) by the gradual removal of lignin at $\mathrm{pH} 4$ and boiling for boiling for 2 hours, the substrate to liquor ratio was maintained at 1:50 and further treated with Sodium bisulphate $5 \%$ w/v. The hollocellulose was treated with $\mathrm{NaOH} 17.5 \%$ filtered, washed with distilled water and dried at $60^{\circ}$ C. (Chattopadhyay and Sarkar 1946: Sarkar et al. 1948)

\section{Pre-treatment of cellulose}

The extracted cellulose was subjected to pre- treatments before hydrolysis. The cellulose was added to $5 \mathrm{M} \mathrm{NaOH}$ solution $(250 \mathrm{ml})$ and heated at $80^{\circ} \mathrm{C}$ for 3 hours. The slurry was then filtered and washed thoroughly with distilled water until till neutral $\mathrm{pH}$. After drying at $105^{\circ} \mathrm{C}$, the cellulose treated with DMSO $(250 \mathrm{ml})$ at $80^{\circ} \mathrm{C}$ in a water bath for 3 hours. It was then filtered, washed with distilled water $(250 \mathrm{ml})$ and dried.

\section{Synthesis and purification of nanocellulose}

After the pre-treatment, the cellulose was transferred in to an acidic solution consisting of (made of $600 \mathrm{ml}$ distilled water, $100 \mathrm{ml} 12.1 \mathrm{~N}$ $\mathrm{HCl}$ and $300 \mathrm{ml} 36 \mathrm{~N} \mathrm{H}_{2} \mathrm{SO}_{4}$ ). The ratio of cellulose to hydrolysate was $1 \mathrm{~g} / 40 \mathrm{ml}$ and this suspension was sonicated for 8 hours. After hydrolysis, the fiber slurry turned in to a milky colloid suspension. The mixture was then centrifuged at $2000 \mathrm{RCF}$ for 15 minutes. After washing the products were neutralized with $2 \mathrm{~N}$ $\mathrm{NaOH}$ and washed with distilled water $(250 \mathrm{ml})$. The sequential process of filtering, washing and centrifuging the products thus obtained was lyophilized. White or ivory yellow coloured fine powder was after lyophilization.

\section{Characterization of cellulose and nano} cellulose from coir pith

To study the nanocellulose extracted from the coir pith, FT-IR analysis using Thermo Nicolet, Avatar 370 model instrument was carried out 64 scans were carried out from 4000$400 \mathrm{~cm}^{-1}$ and resolution was about $0.9 \mathrm{~cm}^{-1}$. DTGS Detector $\left(7800-350 \mathrm{~cm}^{-1}\right)$ was used as a detector. X-ray diffraction (XRD) of the cellulose samples was recorded on Bruker AXS D8 Advance model equipment for highresolution surface imaging at wavelength 1.5406 $\mathrm{A}^{\circ}$. Thermo gravimetric measurements were performed by using a METTER TOLEDO TGA/SDTA851 $1^{\mathrm{e}}$. The surface morphology was studied by using Scanning electron microscope Joel JSM-6380LV at $15 \mathrm{KV}$.

\section{Results and Discussion}

Studies on preparation of spherical nanocrystal cellulose (Xiao-fang Li et al 2010) have prepared nanocrystal cellulose from natural cotton fibre. Zang et al in 2007 have reported a practical procedure for synthesising cellulose nanospheres from cellulosic fibres. In the present study studies were carried on coir pith, which has $28 \%$ of cellulose and can be a potential substrate for the production of nanocellulose. During the extraction of cellulose, the lignocelluloscic biomass was treated with sodium chlorite to remove the lignin. The hollocellulose ( $\alpha$-cellulose + hemicelluloses) and was treated with sodium hydroxide leading to the solubilisation of hemicelluloses and leaving behind the $\alpha$-cellulose. The SEM image of cellulose extracted from coir pith is shown in the Figure 1.

Nanocellulose was prepared by the acid hydrolysis of cellulose. Acid hydrolysis was carried out in a heterogeneous system and depends on the pre-treatment process, mixed acid concentration, hydrolysis time and other factors. Sodium hydroxide $(5 \mathrm{~N})$ treatment of cellulose at $80^{\circ} \mathrm{C}$ for 3 hours was followed by treatment with DMSO results in the initial swelling of cellulose. The purpose of pretreatment process was for increased hydrolysate penetration in to the cellulose fibre. The 
Cord 2012, 28 (1)

Figure 1. SEM image of obtained cellulose

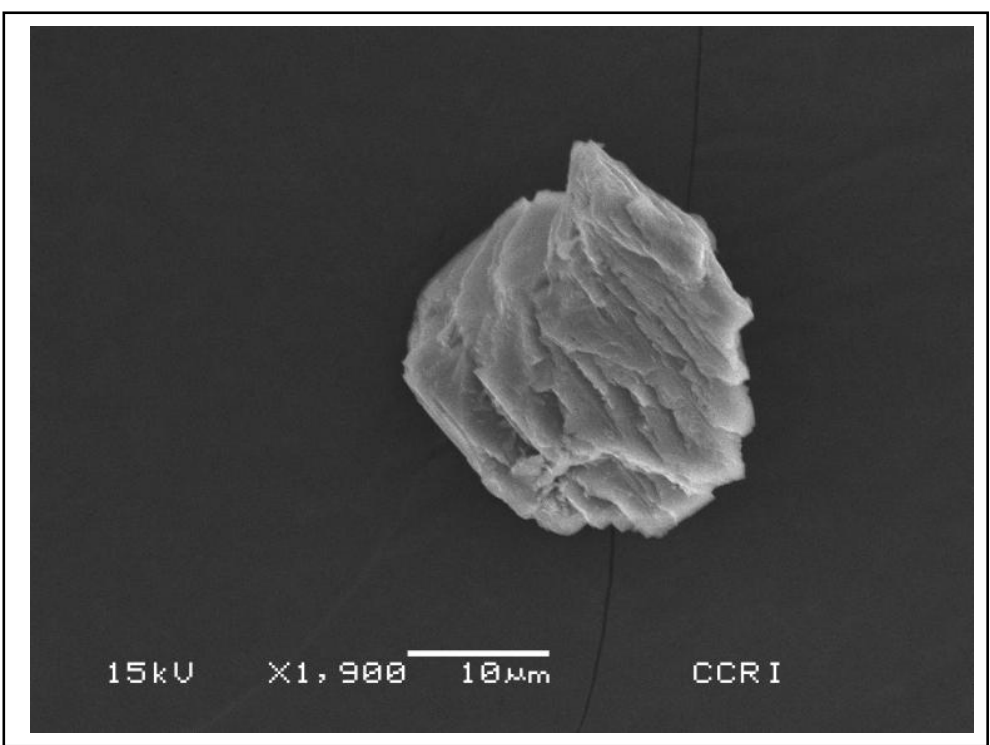

Figure 2. FT-IR spectra of cellulose extracted from coir pith

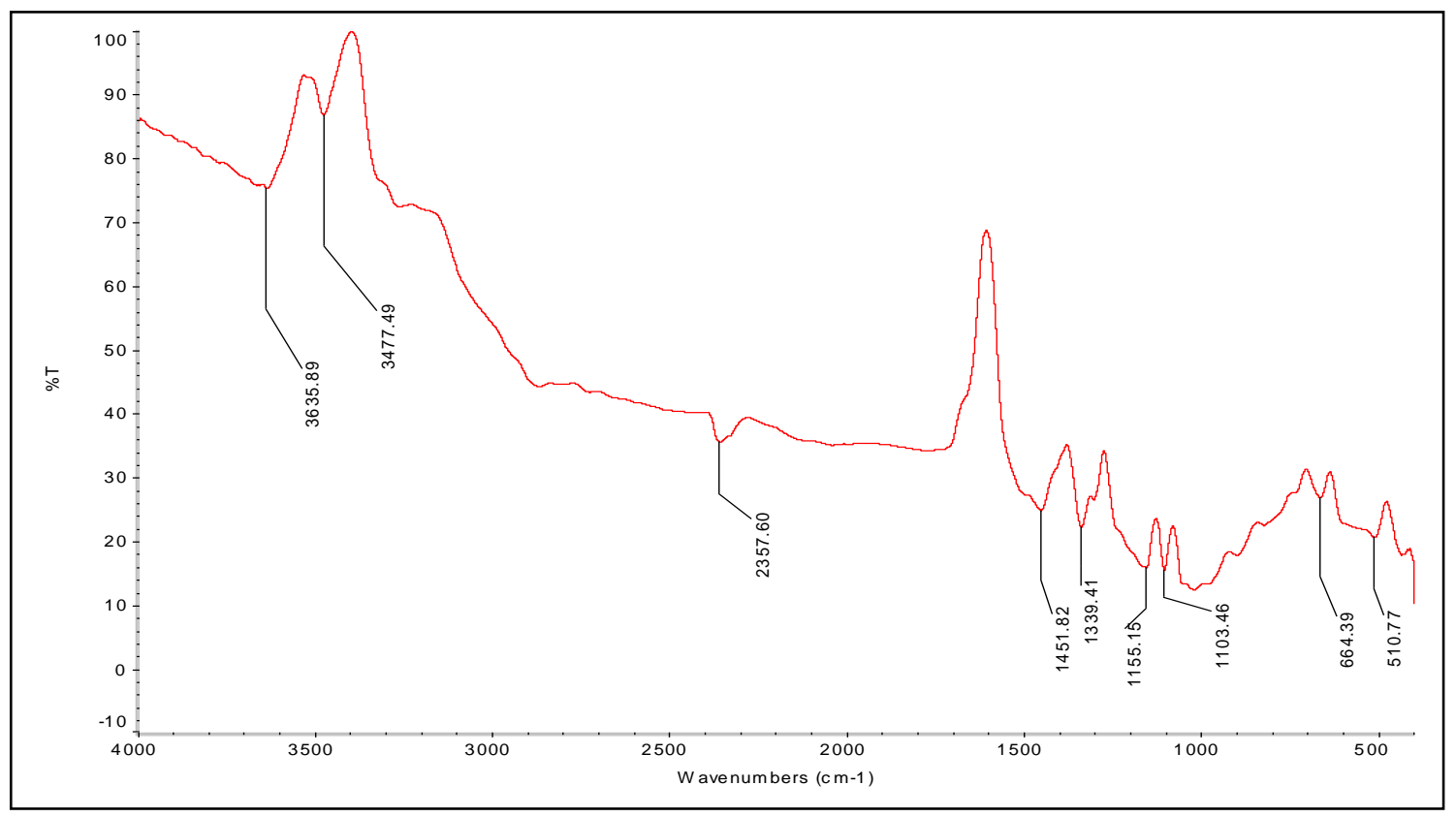


degradation of cellulose to nanometre dimensions occurs only under strong hydrolysis conditions. To avoid the complete hydrolysis of cellulose to glucose or carbonization mild conditions are applied. Mixtures of $\mathrm{HCl}-\mathrm{H}_{2} \mathrm{SO}_{4}$ solution in ratio proved to be a better choice, since it is to avoid such extremes. It is very difficult to control the hydrolysis degree at high temperatures; the reactions were carried at moderate temperature. Acid ultrasonic treatment plays a significant role to control the size of nanocellulose.

\section{Characterisation of cellulose}

The most convenient method for the elucidation of structural features of cellulose is FT-IR spectroscopy and is shown in figure 2 . The broad bands appear around $3000-3700 \mathrm{~cm}^{-1}$ which corresponds to the stretching region of $\mathrm{OH}$ groups. In the present study the FT-IR spectrum, displayed peaks at $3624.11 \mathrm{~cm}^{-1}$, $3477.49 \mathrm{~cm}^{-1}$. The hydroxyl band at 3477.49 $\mathrm{cm}^{-1}$ is the characteristic of the stretching vibration of cellulose hydroxyls bonded to carbons 2, 3, and 6 of the glucose (Van Dam et al.2004b; Joseph et al.2002). The broadening of this band, depends on the chemical treatment and to changes of theinter and intra molecular hydrogen bonding in Polysaccharides (Van dam et al, 2004). The Pyranose ring skeletal lies in the range $1170-1082 \mathrm{~cm}^{-1}$, the absorption band observed at $1155.15 \mathrm{~cm}^{-1}$ is representative of the anti symmetric bridge stretching of C-O-C groups in the cellulose. The inplane ring stretching gives a slight shoulder at $1103 \mathrm{~cm}^{-1}$. The band at $1451 \mathrm{~cm}^{-1}$ is assigned to the symmetric $\mathrm{CH}_{2}$ bending and the band at 1339 $\mathrm{cm}^{-1}$ is ascribed to $\mathrm{CH}_{2}$ wagging vibrations in cellulose. (Colthup et al, 1990, yadav, 2005). The band appearing at $664.39 \mathrm{~cm}^{-1}$ corresponded to the out -of plane bending of $\mathrm{C}-\mathrm{OH}$. (Liu et al.2006; Oh et al. 2005; Fengel et al.1991; Wiley et al.1987).

\section{Thermogravimetric Analysis}

The thermogravimetric analysis permits to study the decomposition of the obtained cellulose and nanocellulose, from this data we can predict the thermal stability of the cellulose obtained. The data was compared to the standard commercially available cellulose (Juan I. Moran. Vera et al). TGA of standard cellulose, extracted cellulose and nanocellulose are shown in figures 3,4 and 5 respectively. A slight weight loss can be seen in the range of $25-120^{\circ} \mathrm{C}$ which is attributed to the evaporation of the humidity of the material.

In the thermal analysis, cellulose decomposition started at $315^{\circ} \mathrm{C}$ and persisted until $400^{\circ} \mathrm{C}$ (Yang et al. 2007). In the commercial cellulose the sharp weight loss starts at $315^{\circ} \mathrm{C}$ and continued up to $400^{\circ} \mathrm{C}$. The decomposition of cellulose obtained from the coir pith starts at $250^{\circ} \mathrm{C}$ and continued up to $400^{\circ} \mathrm{C}$. In the case of nanocellulose the decomposition starts at $300^{\circ} \mathrm{C}$ and continued up to $400^{\circ} \mathrm{C}$. The $10 \% \mathrm{~T}_{10}$ and $50 \% \mathrm{~T}_{50}$ temperature decomposition occurred at of $160^{\circ} \mathrm{C}$ and $335^{\circ} \mathrm{C}$ and that of nanocellulose $\mathrm{T}_{10}$ was $190^{\circ} \mathrm{C}$ and $\mathrm{T}_{50}$ was $340^{\circ} \mathrm{C}$. The increasing trend of decomposition temperature indicates that the thermal stability of nanocellulose is higher than that of the cellulose obtained from the coir pith which in turn shows that during the nano cellulose production the crystallinity of the nano cellulose increased which results in the increase in the thermal stability of nano cellulose.

\section{XRD analysis of cellulose and nanocellulose from coir pith}

In order to predict the crystallinity and size of the nano cellulose, X-ray diffraction analysis was carried out. The XRD of commercial cellulose is shown in the figure 6 . The crystallinity index (CI) of the cellulose was estimated by means of the following equation (Mwaikamb and Ansell, 2002).

$$
\begin{aligned}
& \mathrm{CI}=\mathrm{I}_{(002)}-\mathrm{I}_{(\mathrm{am})} \mathrm{X} 100 . \\
& \mathrm{I}_{(002)}
\end{aligned}
$$

$I_{(002)}$ is the peak intensity of $2 \Theta$ angle close to $26^{\circ}$ representing crystalline material and $\mathrm{I}_{(\mathrm{am})}$ is the counter reading at peak intensity at a $2 \Theta$ angle close to $18^{\circ}$ representing amorphous material in the cellulose. X-ray diffraction of commercial cellulose shows the peak in the range $20-30^{\circ}$. 
Cord 2012, 28 (1)

Figure 3. TGA of commercial cellulose

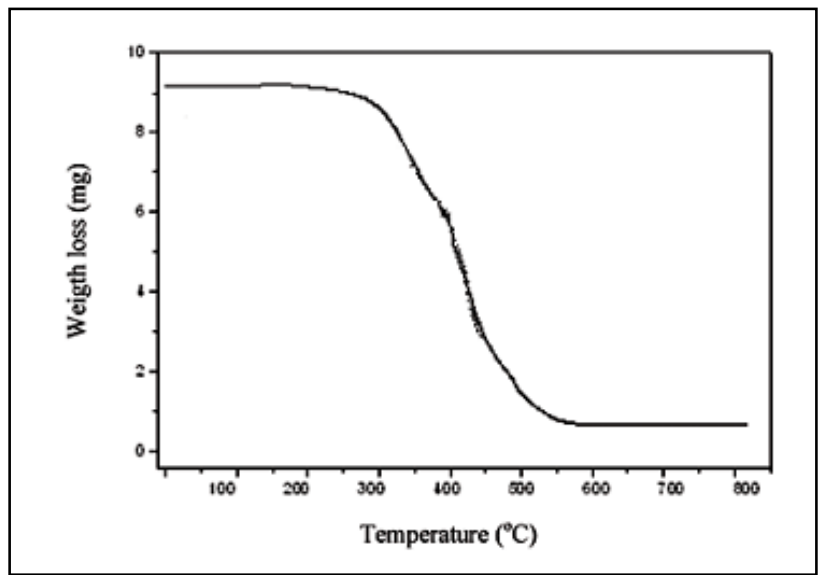

Figure 4. TGA of cellulose extracted from coir pith

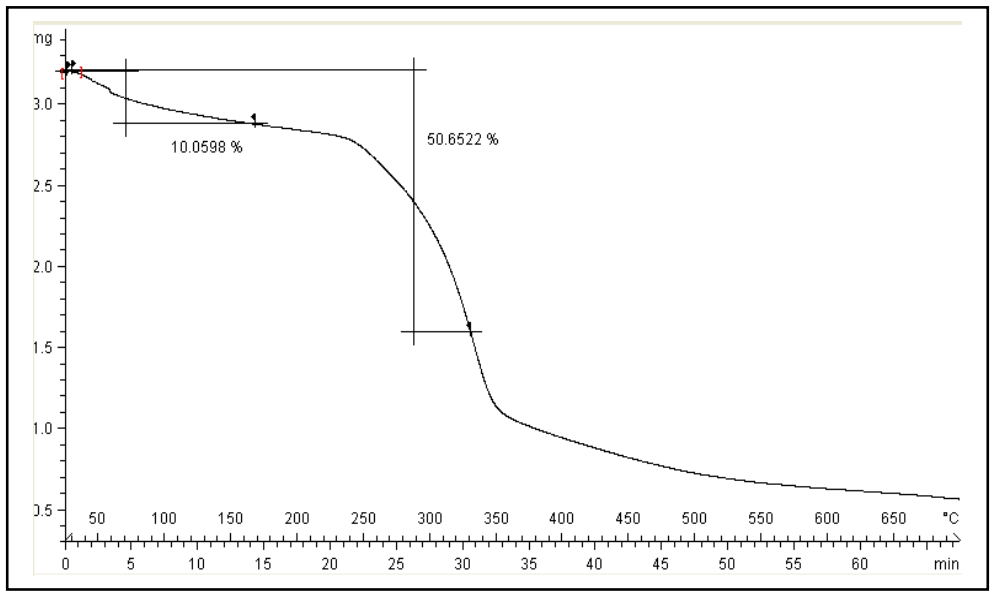

Figure 5. TGA of nanocellulose

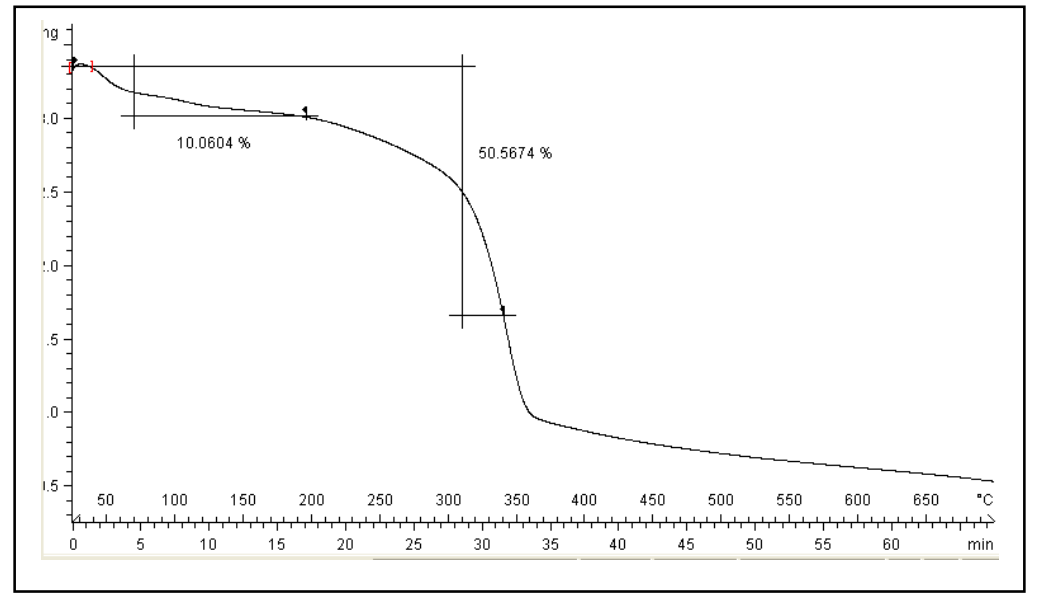


Cord 2012, 28 (1)

Figure 6. XRD of commercial cellulose

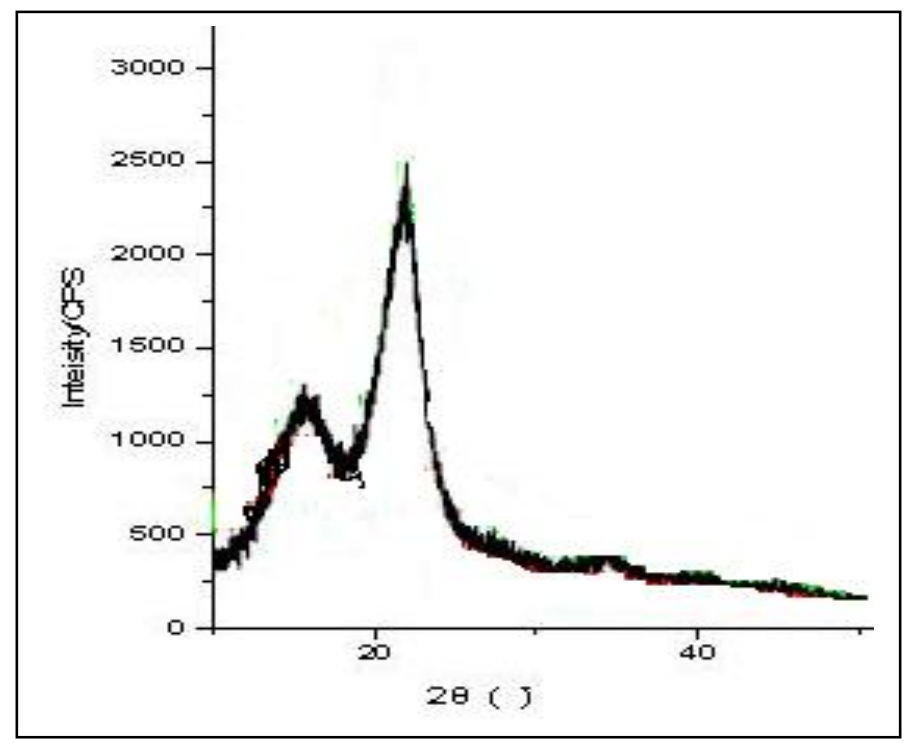

Figure 7. XRD of cellulose extracted from the coir pith

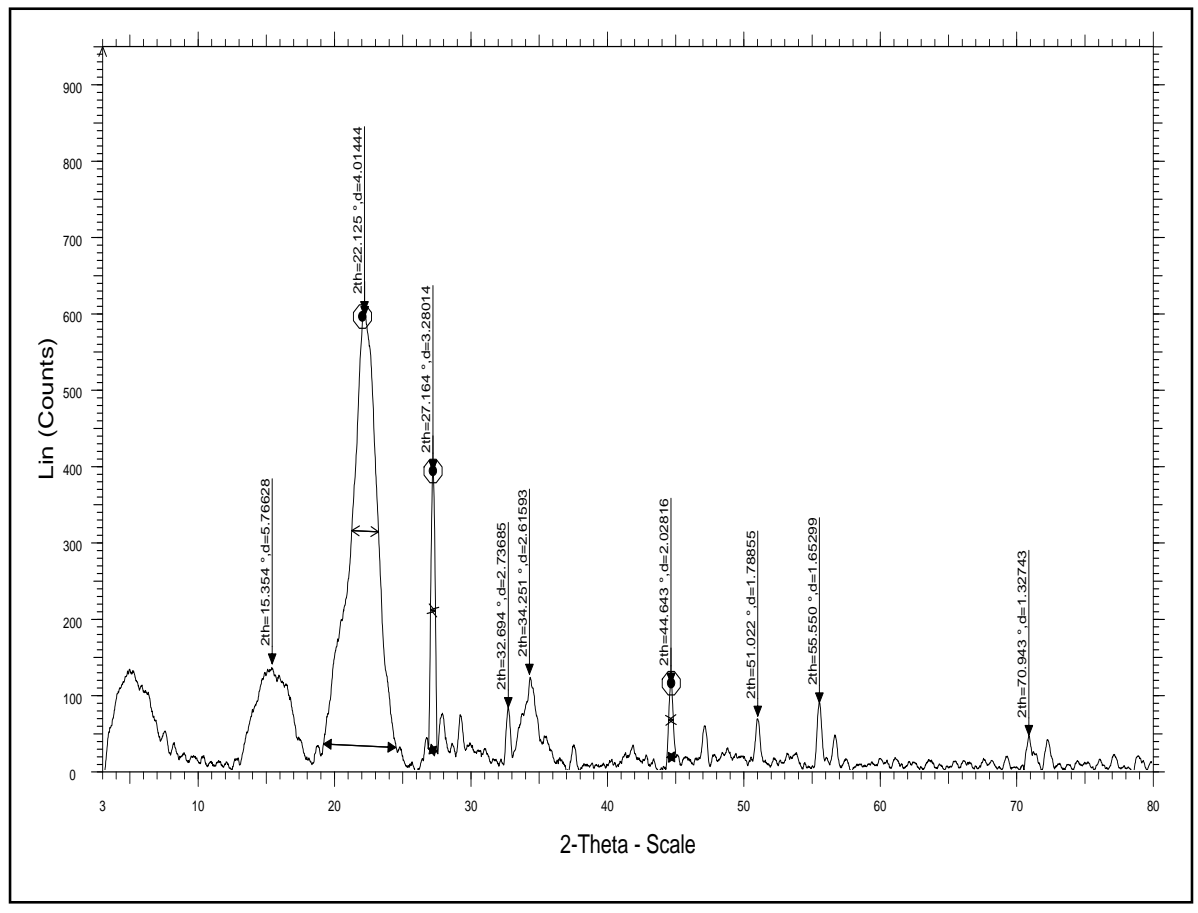


Cord 2012, 28 (1)

Figure 8. XRD of nanocellulose obtained from the coir pith

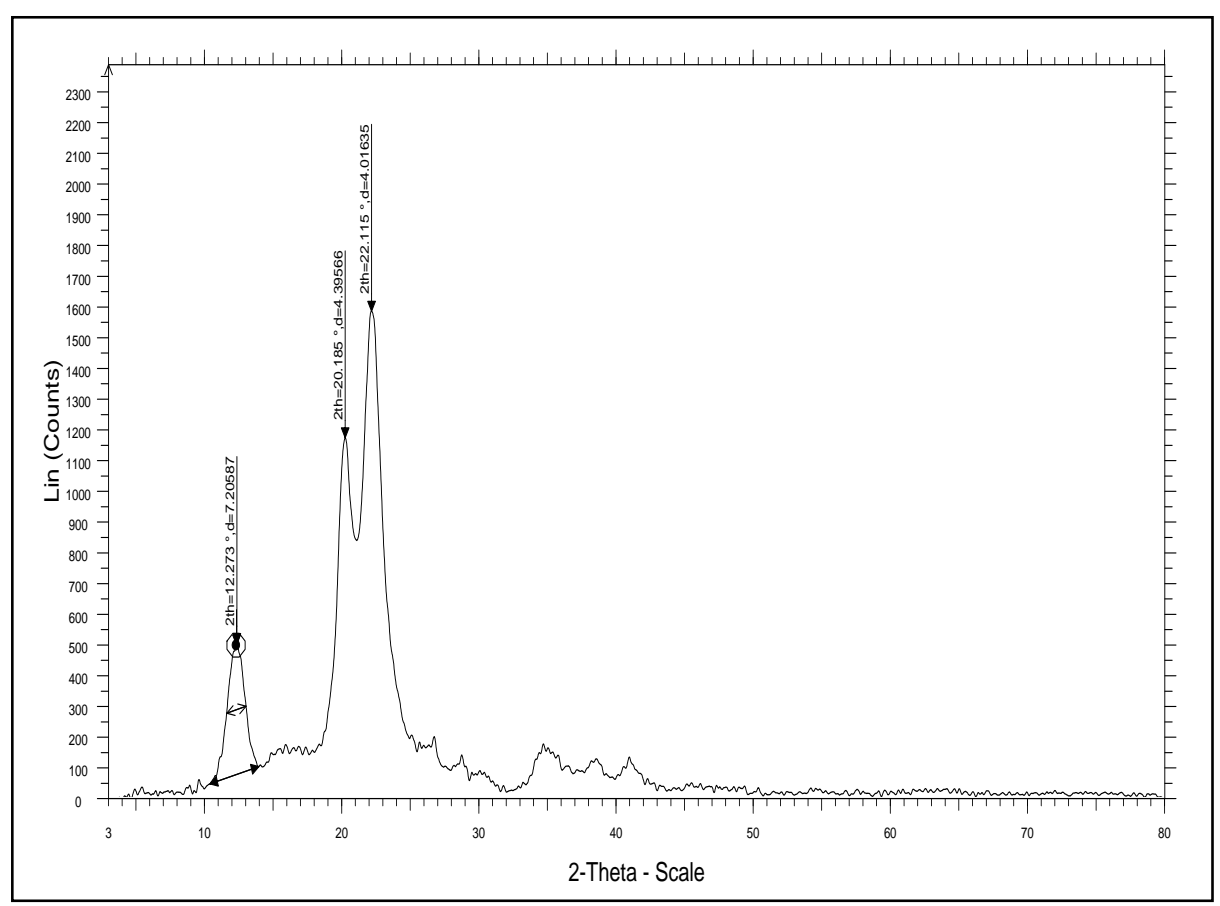

Figure 9

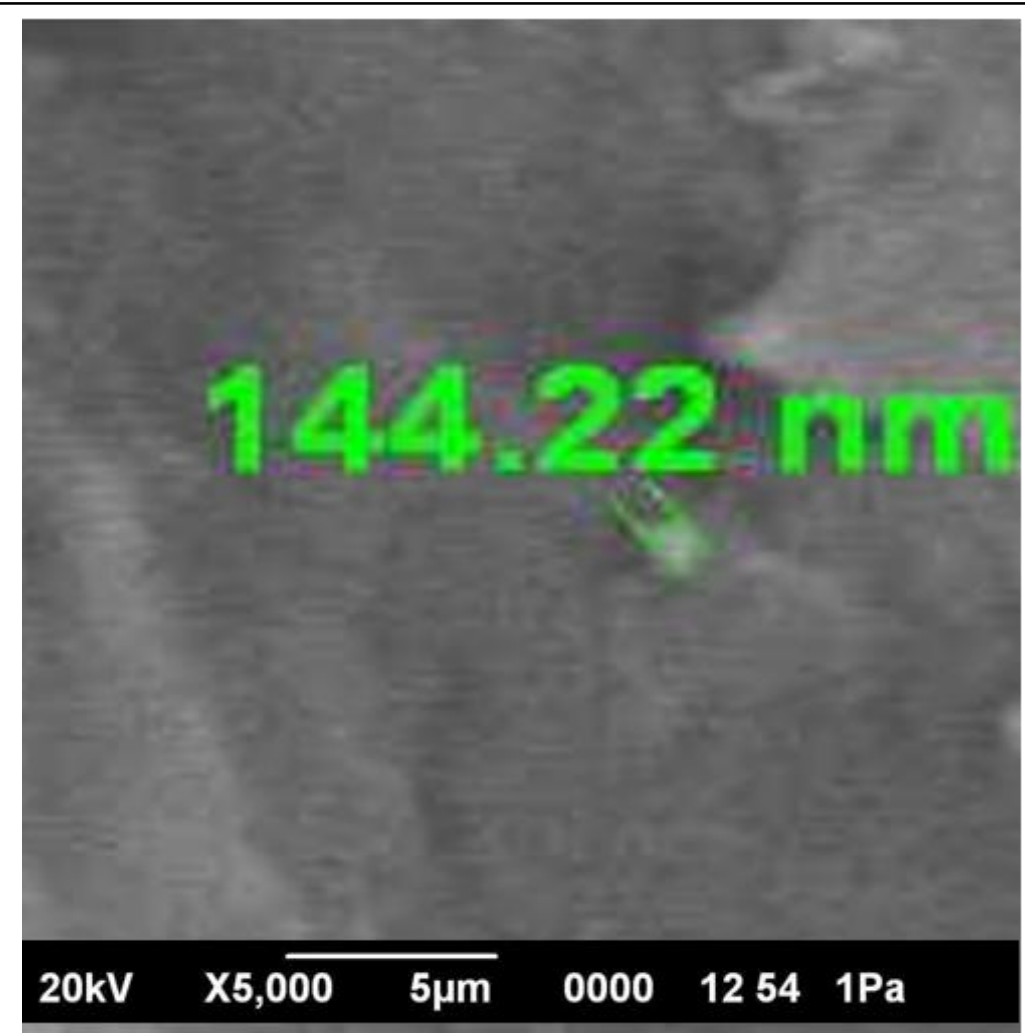


By comparing the results with standard graph of commercial cellulose, the X-ray diffraction pattern of the cellulose extracted from the coir pith (Figure 7) shows the peak in the same range. The peak intensity of $2 \Theta$ angle lies close to $22.125^{\circ}$ representing crystalline material and $I_{(a m)}$ is the counter reading at peak intensity at a $2 \Theta$ angle lying close to $15.354^{\circ}$ representing amorphous material in the cellulose. The CI of the cellulose extracted from the coir pith was observed to be 30.6.

From observation of the X-ray diffraction pattern of nanocellulose, a decrease of diffraction intensities was seen in the amorphous region of nanocellulose around $18^{\circ}$ and a doublet in the range $20-30^{\circ}$ which clearly indicates that the sample has a crystal lattice of the cellulose I and II. The CI of cellulose I and cellulose II were found to be 39.2 and 44.5. From the CI values it could be confirmed that the CI value of nanocellulose is greater than that of the CI of the cellulose extracted from the coir pith. Average size calculated by using Scherer formula.

$$
\mathbf{D}=\frac{\mathbf{0 . 9} \hat{\mathbf{a}}}{\boldsymbol{\beta} \operatorname{Cos} \boldsymbol{\theta}} \begin{aligned}
& \hat{\mathbf{a}}=\text { wavelength }\left(1.5406 \mathrm{~A}^{\circ}\right), \\
& \boldsymbol{\beta}=\text { extent of broadening }
\end{aligned}
$$

The average crystalline size of cellulose I and cellulose II part was observed to be 1.836 and $2.016 \mathrm{~nm}$ respectively. The yield of nanocellulose was observed to be $42 \%$ with respect to the cellulose extracted from the coir pith.

\section{SEM characterization of nanocellulose}

SEM images of the nanocellulose from coir pith are shown in the Figure 9 and showing $144.22 \mathrm{~nm}$ in size.

\section{Conclusion}

The present study demonstrates that coir pith is an excellent substrate for the production of nanocellulose through extraction of cellulose from coir pith and followed by acid hydrolysis process. Finally, mixed crystallinity crystal form of cellulose I and II can be obtained and is characterized by XRD, thermal gravimetric analysis and SEM. Coir pith is considered as a pollutant, conversion of same to nanocellulose results in the preparation of new-nano composites materials and can be used for biomedical applications. NCC production would be a promising product in the future and a boon to the entire coir industry.

\section{Acknowledgment}

Ms. Subha P.V thanks Coir Board, for providing funds for carrying out this research work. Thanks are due extend to Mr. V. S. Vijayaraghavan, Chairman, Coir Board, and Dr. U. S. Sarma, Director, CCRI for their keen interest and continuous guidance in the work.

\section{Reference}

Jiangauo Zang, Thomas J. Elder, Yunqiao Pu, Arthur J. Ragauskas., Facile synthesis of spherical cellulose nano particles., Carbohydrate Polymers., 2007, 607-611.

Juan I. Morian, Vera A, Alvarez, Viviana P, and Cyras., Extraction of cellulose and preparation of nano cellulose from sisal fibers. Cellulose., 2008, 15, 149-159.

Klemm, D., Schmauder, H., and Heinze, T. Cellulose., Biopolymers., 6, 275-319

Li, X., Ding, E., and Li, G., A method of preparing spherical nano crystal cellulose with mixed crystalline forms of cellulose II and I., Chinese Journal of polymer Science., 2001, 19 (13), 291-296.

Loeloich, M., and Leykin, A., "Nano-cellulose and its application", J.SITA., 2004, 6(3), 17-24.

Michael loelovich., Cellulose as a nanostructured polymer: A short review., BioResources, 2008, 3(4), 1403-1418.

Mehdi Jonoobi, Jalaluddin Harun, Paridah Md. Tahir, Lukmanul Hakim Zaini, Syeed SaifulAzry, and Majid Davoodi Makinejad., Charecteristics of nanofibers extraction from Kenaf Core. BioResources, 2010, 5(4), 2556-2566.

Oksman, K and Sain M., "Introduction to cellulose nanocomposites" Cellulose Nano composites; Processing, Characterization 
and Properties, ASC Symposium Series 938, Oxford Press, 2006, 2-8.

Samir, M.A.S.A., Alloin, F., Dufresne, A., Review of recent research in to celluloscic whiskers, their properties and their application in nanocomposites field. Biomacromolecules, 2005, 6(2), 612-626.

Youssef Habibi, Lucian A. Lucia and Orland J. Rojas., Cellulose Nanocrystals: Chemistry, Self-Assembly, and Applications, American Chemical Society., 2010, 110, 3479-3500. 